Therapeutics, Employee of: Formerly Amgen Inc.; Partner in the Cascadia Drug Development Group, J. Merrill Consultant for: EMD Serono, BristolMyerSquibb, Human Genome Sciences, UCB, AstraZeneca, Celgene, J. Chung Shareholder of: Amgen Inc., Employee of: Amgen Inc.

DOI: 10.1136/annrheumdis-2017-eular.1127

\section{OP0235 A NOVEL B CELL SPECIFIC IFN-I BIOMARKER IS ASSOCIATED WITH PLASMABLAST NUMBERS FOLLOWING B CELL DEPLETION THERAPY IN SLE}

Y.M. El-Sherbiny ${ }^{1,2}$, M.Y. Md Yusof ${ }^{1,2}$, E. Hensor ${ }^{1,2}$, A. Rawstron ${ }^{3}$, M. Wittmann ${ }^{1,2}$, P. Emery ${ }^{1,2}$, E.M. Vital ${ }^{1,2}{ }^{1}$ Leeds Institute of Rheumatic and Musculoskeletal Medicine, University of Leeds: ${ }^{2}$ National Institute of Health Research Leeds Musculoskeletal Biomedical Research Unit; ${ }^{3}$ Haematological Malignancy Diagnostic Service, Leeds Teaching Hospitals NHS Trust, Leeds, United Kingdom

Background: SLE is a Type I interferon (IFN-I) mediated disease with autoreactive B cells. Plasmablasts, the immediate progeny of B cells, are expanded in SLE and correlate with disease activity. We showed that their rate of regeneration after therapeutic B cell depletion with rituximab is variable and predicts relapse[1]. IFN-I has been shown in vitro to induce the differentiation of $B$ cells into plasmablasts. We previously showed that therapeutic B cell depletion with anti-CD20 mAb leads to a transient reduction in CD20-negative plasmablasts, following which plasmablasts repopulate and their numbers predict clinical relapse. We developed tetherin as a flow cytometric, cell-specific marker for IFN-I response.

Objectives: To test the hypothesis that memory B cell tetherin determines the rate of plasmablast repopulation after rituximab.

Methods: 117 rituximab-treated SLE patients were studied prosectively using BILAG-2004 and flow cytometry. In 97 responders we tested plasmablasts at 6 months as a predictor of clinical relapse before 12 months to validate our previous finding. In 50 patients pre-rituximab and 28 patients post-rituximab we performed additional flow cytometry to measure tetherin on each cell subset. Expression of 18 ISGs was measured using Taqman on PBMCs and an ISG score calculated. Results: We divided clinical responders to rituximab into earlier relapse $(12$ months) or later relapse (>12 months). As in our published discovery cohort, plasmablasts were strongly predictive of clinical relapse. ROC analysis indicated that a plasmablast count of $>0.0008 \times 10^{9} / \mathrm{L}$ at 6 months yielded $73 \%(95 \% \mathrm{Cl}$ $45-92 \%)$ sensitivity and $90 \%(95 \% \mathrm{Cl} 56-99 \%)$ specificity in predicting earlier relapse; area under the curve of 0.86

Plasmablast numbers after rituximab were associated with Memory $B$ cell tetherin $(\mathrm{R}=0.38, \mathrm{p}=0.047$ ) but not ISG score ( $\mathrm{R}=0.24, \mathrm{p}=0.219)$ (Table 1 ).

In Pre-rituximab there was no relationship between any IFN assay and plasmablast count. After rituximab treatment there was no correlation between plasmablast count and ISG expression, nor with monocyte or NK cell surface tetherin. However, memory B cell tetherin MFI was positively correlated with plasmablast count (Table 1).

Table 1

\begin{tabular}{lcc}
\hline Interferon assay & \multicolumn{2}{c}{ Plasmablast Count (cells $\left.\times 10^{9} / \mathrm{L}\right)$} \\
\cline { 2 - 3 } & Pre-rituximab $(n=50)$ & Post-rituximab $(n=28)$ \\
\hline ISG expression Score: & $-0.11, P=0.448$ & $0.24, P=0.219$ \\
Tetherin protein level: Monocytes & $-0.08, P=0.592$ & $0.20, P=0.296$ \\
T-cells & $-0.16, P=0.269$ & $0.32, P=0.096$ \\
NK cells & $-0.14, P=0.324$ & $0.05, P=0.795$ \\
Naïve B-cells & $-0.04, P=0.801$ & $0.30, P=0.121$ \\
Memory B-cells & $0.07, P=0.618$ & $0.38, P=0.047$ \\
\hline
\end{tabular}

Values are Spearman's Rank Correlation Coefficient and $P$ values.

Conclusions: Although interferon stimulated gene expression is commonly used to measure IFN-I activity, tetherin provides a cell-specific assay. We demonstrate that by measuring IFN-I response in B cells specifically, we could explain plasmablast differentiation, and thereby clinical outcome. Memory B cell tetherin is valuable to immunophetype SLE.

References:

[1] Vital et al. Arthritis Rheum. 2011 Oct;63(10):3038-47.

Disclosure of Interest: None declared

DOI: 10.1136/annrheumdis-2017-eular.6423

\section{OP0236 BENEFIT AND SAFETY OF ANTITHROMBOTIC TREATMENT IN 264 PREGNANCIES IN PATIENTS WITH ANTIPHOSPHOLIPID SYNDROME}

C.M. Yelnik ${ }^{1}$, M. Lambert ${ }^{1}$, E. Drumez ${ }^{2}$, V. Le Guern ${ }^{3}$, J.-L. Bacri ${ }^{4}$, M. Guerra ${ }^{5}$, C.A. Laskin ${ }^{6}$, W. Branch ${ }^{7}$, L.R. Sammaritano ${ }^{5}$, N. Morel ${ }^{3}$, G. Guettrot-Imbert $^{3}$, D. Launay ${ }^{1}$, E. Hachulla ${ }^{1}$, P.-Y. Hatron ${ }^{1}$, J.E. Salmon ${ }^{5}$,

N. Costedoat-Chalumeau ${ }^{3} .{ }^{1}$ Internal Medicine Department, University of Lille, UFR Medicine, Universitary Hospital Center of Lille; ${ }^{2}$ Biostatistics Department, University of Lille, EA 2694. Universitary Hospital Center of Lille, lille: ${ }^{3}$ Internal Medicine Department, Paris Descartes-Sorbonne Paris Cité University, INSERM U1153, Cochin Hospital, Paris; ${ }^{4}$ Internal Medicine Department, Hospital Center of Valenciennes, Valenciennes, France ${ }^{5}$ Rheumatology, Hospital for Special
Surgery, New York City, United States; ${ }^{6}$ University of Toronto and LifeQuest Center for Reproductive Medicine, Toronto, Canada; ${ }^{7}$ University of Utah and Intermountain Healthcare, Salt Lake City, United States

Background: The management of pregnancy in patients with antiphospholipid syndrome (APS) with aspirin and heparin is based on empiric recommendations. Objectives: Our study aimed to evaluate the outcomes of treated patients with thrombotic and obstetric APS and the safety of antithrombotic treatments prescribed during pregnancy.

Methods: Inclusion criteria were (1) APS (Sydney criteria), (2) live pregnancy at 12 weeks of gestation (WG) with (3) follow up data until 6 weeks post-partum. Data were collected prospectively (PROMISSE study) and retrospectively (four French centers). Adverse pregnancy outcomes (APOs) were defined by fetal death or neonatal death; pre-term delivery before 36 WG due to preeclampsia or placental insufficiency; or small for gestational-age (SGA; $<5$ th percentile). Major bleeding was defined as blood loss greater than $500 \mathrm{~mL}$ and/or requiring surgery or transfusion.

Results: 264 pregnancies (87 collected prospectively) in 204 patients were included ( $46 \%$ with a history of thrombosis, and $23 \%$ with associated systemic lupus erythematosus). During pregnancy, treatment included heparin $(n=253$; $96 \%)$ and low-dose aspirin ( $n=223 ; 84 \%)$.

The live birth rate was $86 \%$. APOs occurred in $32 \%$, mostly during the 2nd trimester: fetal deaths $11 \%$, SGA $11 \%$, pre-term delivery before 36 WG due to preeclampsia or placental insufficiency $17 \%$. Thirteen maternal thrombotic events occurred in $12(4.5 \%)$ pregnancies. Forty-six maternal hemorrhagic events occurred in $40(15 \%)$ pregnancies (30 events in the post-partum period). Major bleeding was reported in only 6 pregnancies $(2.3 \%)$ and occurred only after delivery. Except for two events, post-partum hemorrhage occurred in the early post-partum before hospital discharge. No maternal death was observed.

Aspirin therapy during pregnancy was the only independent factor associated with a lower risk of APOs (odds ratio: $0.34 ; 95 \% \mathrm{Cl}: 0.15-0.78 ; \mathrm{p}=0.01$ ) in multivariate analysis.

Neither heparin or aspirin alone, nor combined therapy increased the risk of hemorrhage. In the retrospective cohort, emergency caesarian section was the only factor associated with hemorrhagic events during the study period $(53 \%$ hemorrhages in patients who underwent emergency caesarian compared to $18 \%$, $\mathrm{p}=0.005$ ). Independent risk factors for APOs were elevated body mass index and the presence of lupus anticoagulant.

Conclusions: We report a high level of obstetrical complications in conventionallytreated APS pregnancies, and a beneficial effect of addition of aspirin to prevent obstetrical morbidity. Moreover, heparin and aspirin were well tolerated and did not increase risk of hemorrhage.

Disclosure of Interest: None declared

DOI: 10.1136/annrheumdis-2017-eular.1926

\section{OP0237 LIFITEGRAST OPHTHALMIC SOLUTION 5.0\% FOR TREATMENT OF DRY EYE DISEASE: COMBINED EVIDENCE FROM 5 RANDOMIZED CONTROLLED TRIALS}

C. Baudouin ${ }^{1}$, M. Darvish-Zargar ${ }^{2}$, E.J. Holland ${ }^{3}$, C.C. Chan ${ }^{4}$, K.K. Nichols ${ }^{5}$, J. Tauber ${ }^{6}$, A. Raychaudhuri ${ }^{7}$, M. Roy ${ }^{7}$, A. Shojaei ${ }^{7}{ }^{1}$ Centre Hospitalier National d'Ophtalmologie des Quinze-Vingts, Paris, France; ${ }^{2}$ McGill University, Montreal, Canada; ${ }^{3}$ Cincinnati Eye Institute, Edgewood, United States;

${ }^{4}$ University of Toronto, Toronto, Canada; ${ }^{5}$ University of Alabama School of Optometry, Birmingham; ${ }^{6}$ Tauber Eye Center, Kansas City; ${ }^{7}$ Shire, Lexington, United States

Background: Dry eye disease (DED) is a multifactorial disease of the tear film and ocular surface, characterized by ocular discomfort and visual disturbance. DED is associated with a number of systemic autoimmune diseases, particularly rheumatoid arthritis and Sjögren's syndrome. ${ }^{2,3}$ Lifitegrast is a lymphocyte function-associated antigen-1 (LFA-1) antagonist that inhibits T-cell-mediated inflammation (an underlying factor in DED) and is approved in the US for the treatment of signs and symptoms of DED (lifitegrast ophthalmic solution $5.0 \%$, Xiidra ${ }^{\circledR}$.

Objectives: To evaluate the combined evidence from 5 clinical trials of lifitegrast ophthalmic solution $5.0 \%$ (LIF) in subjects with dry eye disease (DED).

Methods: Adults with DED were randomized to LIF or placebo (PBO) in 5 randomized, double-masked, placebo-controlled trials: 4 12-week efficacy/safety studies (phase 2, LIF $n=58$, PBO $n=58$; phase 3 trials: OPUS-1, LIF $n=293$, PBO $n=295$; OPUS-2, LIF $n=358$, PBO $n=360 ;$ OPUS-3, LIF $n=355$, PBO $\mathrm{n}=356$ ), and a 1-year safety study (SONATA, LIF $\mathrm{n}=220, \mathrm{PBO} n=111$ ). Individuals with secondary Sjögren's syndrome associated with autoimmune disease (eg, rheumatoid arthritis, systemic lupus erythematosus) were eligible to participate if they were not immunodeficient/immunosuppressed, not taking steroids, and met all other inclusion and exclusion criteria. Change from baseline to day 84 in DED signs and symptoms was evaluated across the 12-week studies. Key measures were inferior corneal staining score (ICSS; 0-4 scale), eye dryness score (EDS; visual analogue scale [VAS], 0-100 scale), and visual-related function subscale of a symptom scale ( $0-4$ scale). Pooled safety data (LIF $n=1287$, PBO $n=1177$ ) from all 5 trials were also analyzed.

Results: LIF improved ICSS versus PBO in the phase 2 study (secondary endpoint; treatment effect 0.35 , nominal $P=0.0209$ ), OPUS-1 (co-primary; 0.24, 
Key efficacy results of lifitegrast ophthalmic solution $5.0 \%$ across 12 -week multicenter randomized controlled trials (ITT population with LOCF)

\begin{tabular}{|c|c|c|}
\hline Study & Symptom endpoint & Sign endpoint \\
\hline Phase 2 & $\begin{array}{l}\text { Visual-related function subscale } \\
\text { (secondary, change from baseline to } \\
\text { day } 84 \text { ): TE } 0.37 \text {, nominal } P=0.0394 \text {. }\end{array}$ & $\begin{array}{l}\text { - Primary endpoint of ICSS at day } 84 \\
\text { not met. } \\
\text { - ICSS (secondary, change from } \\
\text { baseline to day } 84 \text { ): TE } 0.35 \text {, nominal } \\
P=0.0209 \text {. }\end{array}$ \\
\hline OPUS-1 & $\begin{array}{l}\text { - Coprimary endpoint of change from } \\
\text { baseline to day } 84 \text { on visual-related } \\
\text { function subscale not met. }\end{array}$ & $\begin{array}{l}\text { - ICSS (co-primary, change from } \\
\text { baseline to day } 84 \text { ): TE } 0.24 \text {, } \\
P=0.0007 \text {. }\end{array}$ \\
\hline OPUS-2 & $\begin{array}{l}\text { EDS (VAS; co-primary, change from } \\
\text { baseline to day } 84 \text { ): TE } 12.61 \text {, } \\
P<0.0001 .\end{array}$ & $\begin{array}{l}\text { - Coprimary endpoint of change from } \\
\text { baseline to day } 84 \text { in ICSS not met. }\end{array}$ \\
\hline OPUS-3 & $\begin{array}{l}\text { EDS (VAS; primary, change from } \\
\text { baseline to day } 84 \text { ): TE } 7.16 \text {, } \\
P=0.0007 \text {. }\end{array}$ & $\begin{array}{l}\text { - ICSS (ad hoc, change from baseline } \\
\text { to day } 84 \text { ): TE } 0.17 \text {, nominal } \\
P=0.0144 \text {. }\end{array}$ \\
\hline
\end{tabular}

Eye dryness score (EDS; visual analogue scale [VAS]); ICSS, inferior corneal staining score; ITT,

intention-to-treat; LOCF, last observation carried forward; $T E$, treatment effect.

$P=0.0007$ ), and OPUS-3 (ad hoc; 0.17 , nominal $P=0.0144$ ). LIF reduced EDS (VAS) versus PBO in OPUS-2 (co-primary; 12.61, $P<0.0001$ ) and OPUS-3 (primary; 7.16, $P=0.0007$ ). The OPUS-1 co-primary symptom endpoint of visualrelated function subscale, and the OPUS-2 co-primary sign endpoint of ICSS, did not achieve statistical significance. In the pooled safety analysis, total exposure was 415.65 person-years for LIF, and 332.15 person-years for PBO. Adverse events were mostly mild or moderate in severity. There were no serious ocular treatment-emergent adverse events (TEAEs) and withdrawals due to TEAEs were infrequent (LIF, 7.0\%; PBO, 2.6\%).

Conclusions: LIF improved signs and symptoms of DED in adults with DED and appeared to be well tolerated with no serious ocular TEAEs reported.

References:

[1] DEWS. Ocul Surf. 2007;5:75-92.

[2] Fujita M et al. Am J Ophthalmol. 2005;140:808-13.

[3] Patel SJ, Lundy DC. Am Fam Physician. 2002:66:991-8.

Acknowledgements: This study was sponsored by SARcode Bioscience (now a wholly owned subsidiary of Shire PLC) and Shire Development LLC.

Disclosure of Interest: C. Baudouin Consultant for: Alcon Laboratories, Inc., Allergan, Dompé, Horus Pharma, Santen Inc., Thea Pharmaceuticals, M. DarvishZargar Consultant for: Allergan, Novartis, Abbott Medical Optics, E. Holland Grant/research support from: Alcon Laboratories Inc., Allergan, Mati Therapeutics, Omeros, PRN, Senju Pharaceuticals, Consultant for: Alcon Laboratories Inc., Allergan, Bausch \& Lomb, Kala Pharmaceuticals, Mati Therapeutics, Omeros, PRN, RPS, Senju Pharaceuticals, Shire/SARcode, TearLab, TearScience, Speakers bureau: Alcon Laboratories Inc., Allergan, Bausch \& Lomb, Omeros, Senju Pharaceuticals, Shire/SARcode, TearScience, C. Chan Grant/research support from: Allergan, Bausch and Lomb, TearLab, Consultant for: Allergan, Bausch \& Lomb, Alcon Labs Inc., TearScience, K. Nichols Grant/research support from: Kala Pharmaceuticals, Shire PLC, TearScience, Vistakon, Consultant for: Allergan, InSite Vision Inc., Kala Pharmaceuticals, Parion Sciences, Santen, ScienceBased Health, Shire PLC/SARcode, J. Tauber Consultant for: Allergan, Bausch \& Lomb, Shire PLC, Senju Pharaceuticals, Speakers bureau: Shire/SARcode, A. Raychaudhuri Employee of: Shire PLC (at the time of the study), M. Roy Employee of: Shire PLC, A. Shojaei Employee of: Shire PLC

DOI: 10.1136/annrheumdis-2017-eular.5890

\section{LB0002 48 WEEK COMPLETE REMISSION OF ACTIVE LUPUS NEPHRITIS WITH VOCLOSPORIN}

V. Dobronravov ${ }^{1}$, M.A. Dooley ${ }^{2}$, S.A. Haq $^{3}$, I. Adzerikho ${ }^{4}$, O. Bugrova ${ }^{5}$, D. Isenberg ${ }^{6}, F$. Houssiau ${ }^{7}, N$. Solomons ${ }^{8}$, R. Huizinga ${ }^{8}$ on behalf of AURA-LV Study Group. ${ }^{1}$ Pavlov First St. Petersburg State Medical University, St. Petersberg, Russian Federation; ${ }^{2}$ UNC, Chapel Hill, United States; ${ }^{3}$ Bangabandhu Sheikh Mujib Medical University (BSMMU), Dhaka, Bangladesh; ${ }^{4}$ Minsk Regional Clinical Hospital, Minsk, Belarus; ${ }^{5}$ Orenburg Regional Clinical Hospital, Orenburg, Russian Federation; ${ }^{6}$ University College Hospital, London, United Kingdom; ${ }^{7}$ Université catholique de Louvain, Brussels, Belgium; ${ }^{8}$ Aurinia Pharmaceuticals, Victoria, Canada

Background: Voclosporin (VCS) is a novel CNI intended for use in the treatment of autoimmune diseases such as lupus nephritis (LN). VCS's unique structure allows for less pharmacokinetic-pharmacodynamic variability and a potentially improved safety profile compared to other CNls.

Objectives: Achievement of complete remission (CR) as assessed at week 24 (primary objective) and assessment of the efficacy over 48 weeks (secondary objective), with 2 doses of VCS (low dose VCS: $23.7 \mathrm{mg} \mathrm{BID}$ and high dose VCS: $39.5 \mathrm{mg}$ BID) vs. placebo in subjects with active LN.
Methods: The double blind placebo controlled AURA study enrolled 265 subjects with active LN in 20 countries. Patients were randomized into 3 arms (placebo, low dose VCS or high dose VCS) in addition to MMF $2 \mathrm{~g} /$ day and steroids (with rapid tapering). CR was defined as a confirmed urine protein/creatinine ratio (UPCR) of $\leq 0.5 \mathrm{mg} / \mathrm{mg}$ using first morning void and confirmed estimated glomerular filtration rate (eGFR, CKD-EPI equation) $\geq 60 \mathrm{~mL} / \mathrm{min} / 1.73 \mathrm{~m}^{2}$ or no decrease from baseline in eGFR of $>20 \%$ in the presence of low dose steroids. Partial remission (PR) was defined as a $50 \%$ reduction in UPCR. UPCR assessments were made at each visit, together with biomarker data at regular intervals.

Results: We now present the 48 week data showing improved CR rates over the 24 week data. The rate of CR was significantly higher in the low dose VCS compared to the control group (32.6\% vs. $19.3 \%$; OR: $2.03, p=0.045$ ) at 24 weeks. It was $27.3 \%$ in the high dose VCS group ( $p=N S$ ). Both doses of voclosporin demonstrated superiority to control using time to CR, PR $(50 \%$ reduction in proteinuria) and time to PR.

At 48 weeks, $23.9 \%$ of patients on the control arm achieved CR comparted to $49.4 \%$ low dose (OR: $3.21, p<0.001$ ) and $39.8 \%$ high dose (OR: $2.10, p<0.026$ ). Over $92 \%$ of subjects experienced at least one adverse event (AE) with the most common two being infections (58\% low, $66 \%$ high and $55 \%$ placebo) and GI disorders ( $43 \%$ low, $52 \%$ high and $38 \%$ placebo). The overall rate of serious adverse events (SAEs) was numerically higher in both voclosporin groups $(28 \%$ low, $25 \%$ high, $19 \%$ placebo) with the nature of SAEs consistent with those observed in patients with highly active LN. Most deaths occurred in the first 2 months and were: low dose (infection3, ARDS2, thrombotic3, cardiac tamponade, pulmonary hemorrhage), high dose (infection, PE) and control (CVA). All were considered unrelated to drug exposure by the investigators. 3 additional deaths occurred in placebo patients after the conclusion of the 48 weeks of treatment.
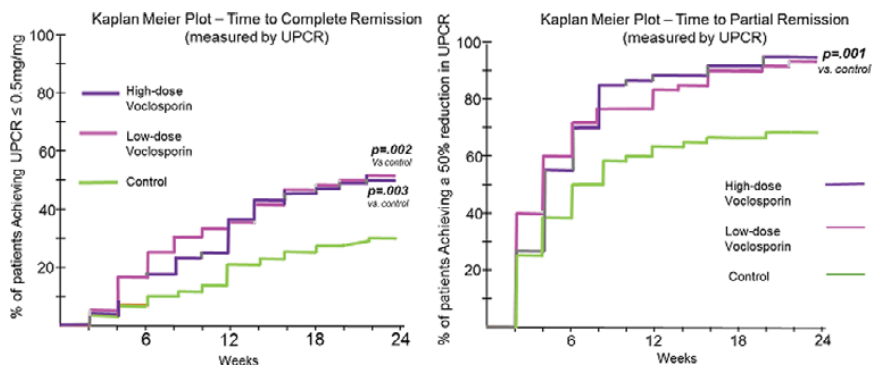

Conclusions: The AURA-LV study is the first global study demonstrating the beneficial effect of VCS, in combination with MMF and steroids, in the treatment of $L N$. Remission rate was rapid. VCS treatment resulted in increasing CR and PR seen by week 6 despite rigorous steroid taper (mean steroid dose $4 \mathrm{mg}$ at week 16). Adverse events were higher in the treated patient group with the nature in keeping with immunosuppression. These promising data will be used to plan subsequent studies of voclosporin in LN.

Acknowledgements: This data was submitted on behalf of the AURA-LV study investigators.

Disclosure of Interest: V. Dobronravov Grant/research support from: Aurinia Pharmaceuticals Inc., M. A. Dooley Grant/research support from: Aurinia Pharmaceuticals Inc., S. A. Haq Grant/research support from: Aurinia Pharmaceuticals Inc., I. Adzerikho Grant/research support from: Aurinia Pharmaceuticals Inc., O. Bugrova Grant/research support from: Aurinia Pharmaceuticals Inc., D. Isenberg: None declared, F. Houssiau: None declared, N. Solomons Shareholder of: Aurinia Pharmaceuticals Inc., Employee of: Aurinia Pharmaceuticals Inc., R. Huizinga Shareholder of: Aurinia Pharmaceuticals Inc., Employee of: Aurinia Pharmaceuticals Inc.

DOI: 10.1136/annrheumdis-2017-eular.7079

\section{FRIDAY, 16 JUNE 2017 Axial spondyloarthritis from risk factor to clinical outcomes}

\section{OP0238 MEASUREMENT OF SPINAL MOBILITY IN AXIAL SPONDYLOARTHRITIS USING INERTIAL SENSORS: RELIABILITY AND VALIDATION PRELIMINARY RESULTS}

F.J. Mata-Perdigon ${ }^{1}$, I.R. Martínez Sanchez ${ }^{2}$, J.L. Garrido-Castro ${ }^{1}$, C. González-Navas ${ }^{1}$, L.M. Fernandez-Ahumada ${ }^{2}$, D.A. Gomez ${ }^{1}$,

V.C. Perez-Guijo ${ }^{1}$, P. Font-Ugalde ${ }^{1}$, E. Collantes-Estevez ${ }^{1} .{ }^{1}$ Maimonides Institute for Biomedical Research of Cordoba; ${ }^{2}$ University of Cordoba, Cordoba, Spain

Background: Axial spondyloarthritis (axSpA) is a chronic rheumatic disease that causes reduction of mobility in the patients' spine. There are several indices to analyze this mobility: BASMI, which lacks precision and sensitivity to change according to different authors, and UCOASMI (1) based on motion capture, which needs extensive resources that limit its practical applicability. Inertial measurement unit sensors (IMU) give, in real time, the 3D orientation of any anatomical place 